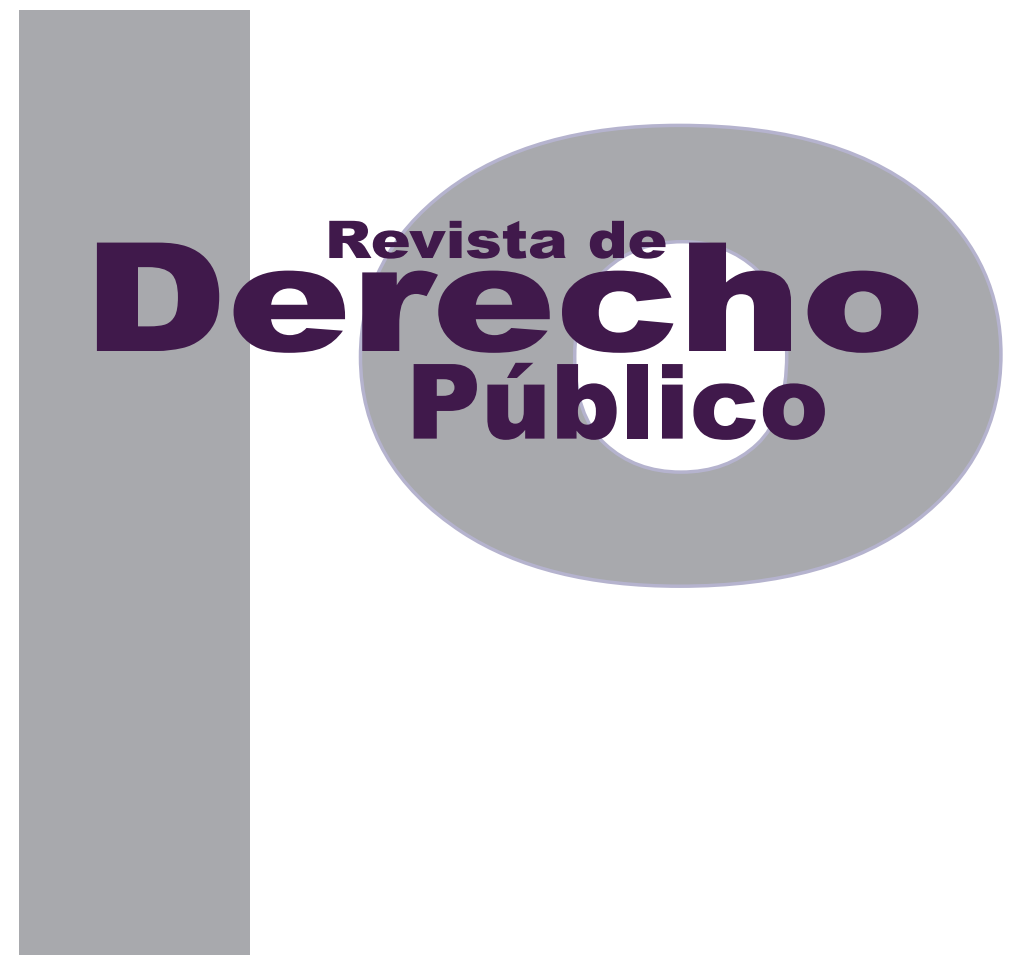

\title{
El MARCO ANALÍtico de HARVARD (GENDER MAINSTREAMING) EN LOS ANÁLISIS DEL DERECHO ECONÓMICO: PROPUESTA PARA VISIBILIZAR A LA MUJER EN LOS PROCESOS DE DESARROLLO ECONÓMICO EN COLOMBIA
}

\author{
LUIS Miguel HoYOS ROJAS
}

Artículo de revisión

DOI: http://dx.doi.org/10.15425/redepub.33.2014.14

Universidad de los Andes

Facultad de Derecho

Revista de Derecho Público N. ${ }^{\circ} 33$

Julio - Diciembre de 2014. ISSN 1909-7778 


\title{
El Marco Analítico de Harvard (gender mainstreaming) en los análisis del derecho económico: propuesta para visibilizar a la mujer en los procesos de desarrollo económico en Colombia
}

\section{Resumen}

Las mujeres colombianas siguen siendo el sector social que ha padecido la situación de desventaja en todo el proceso de desarrollo del país. El hecho es que no se puede desconocer que ellas continúan recorriendo un espinoso camino abonado por la exclusión social, original de los procesos de progreso y modernización económica nacional. Tal vulneración en contra de la mujer colombiana requiere de una propuesta de inclusión de la categoría de género en los análisis del derecho económico como promotor del desarrollo, para demostrar que pese a la existencia de una construcción legal y constitucional que responde al desarrollo armónico de todos y todas, aún persisten temas cardinales inconclusos para su progreso social y económico. Se partirá, entonces, en este documento, de la convicción de que el derecho económico tiene un gran compromiso con los procesos de desarrollo en Colombia y que solamente con la aplicación del Marco Analítico de Harvard (gender mainstreaming) como perspectiva de género se podrán promover interposiciones jurídicas y sociales orientadas a impulsar el bienestar de la mujer colombiana.

Palabras clave: derecho y desarrollo, derecho y género, economía, gender mainstreaming, género.

\section{The Analytical Framework of Harvard "gender mainstreaming" in economic analysis of law: proposal for visibility of women in the process of economic development in Colombia}

\begin{abstract}
Colombian women continue to be the social sector that has suffered from the situation of disadvantage in the process of development of Colombia. The fact is that it cannot be ignored that they are still touring a thorny road paid from the social exclusion, original from the progress and the country's economic modernization processes. Such violations against colombian women, require the existence of a proposal for the inclusion of the category of gender in the analysis of the economic law as a promoter of development, to demonstrate that despite the existence of a legal and constitutional construction which responds to the harmonious development of everyone, there are cardinal issues inconclusive for women's economic and social progress yet. In this sense, this document will depart from the conviction that the economic right has a great commitment to the processes of development in Colombia and that only with the application of the analytical framework of Harvard (gender mainstreaming) as gender mainstreaming in Colombia, legal and social oriented interpositions may promote the well-being of Colombian women.

Keywords: law and development, law and gender, economics, Gender Mainstreaming and gender.

\section{o Marco Analítico de Harvard (gender mainstreaming) nas análises do direito econômico: proposta para visibilizar a mulher nos processos de desenvolvimento econômico na Colômbia}

\section{Resumo}

As mulheres colombianas seguem sendo o setor social que tem padecido da situação de desvantagem em todo o processo de desenvolvimento do país. 0 fato é que não podemos desconhecer que elas continuam percorrendo um caminho espinhoso abonado pela exclusão social, originária dos processos de progresso e modernização econômica nacional. Tal vulnerabilidade contra a mulher colombiana requer uma proposta de inclusão da categoria de gênero nas análises do direito econômico como promotor do desenvolvimento, para demostrar que por mais que exista uma construção legal e constitucional que responde ao desenvolvimento harmônico de todos e todas, ainda persistem temas cardinais, inclusive para seu progresso social e econômico. Neste documento partiremos então da convicção de que o direito econômico tem um grande compromisso com os processos de desenvolvimento na Colômbia e que somente com a aplicação do Marco Analítico de Harvard o gender mainstreaming como perspectiva de gênero poderão ser promovidas interposições jurídicas e sociais orientadas a impulsar o bem-estar da mulher colombiana.

Palavras-chave: direito e desenvolvimento, direito e gênero, economia, gender mainstreaming, gênero. 


\title{
El Marco Analítico de Harvard (gender mainstreaming) en los análisis del derecho económico: propuesta para visibilizar a la mujer en los procesos de desarrollo económico en Colombia*
}

\author{
Luis Miguel Hoyos Rojas*
}

\section{SUMARIO}

Introducción - I. ASPECTOS HISTÓRICOS DEL DERECHO ECONÓMICO, DEL DESARROLLO ECONÓMICO Y LA PERSPECTIVA DE GÉNERO EN COLOMBIA - A. Derecho, desarrollo y mujer en Colombia - B. La promesa incumplida y el nuevo paradigma del derecho económico - II. EL MARCO ANALÍTICO DE HARVARD (GENDER MAINSTREAMING) EN LOS ANÁLISIS DEL DERECHO ECONÓMICO EN COLOMBIA - A. Necesidad de la teoría y perspectiva de género en los procesos de desarrollo económico auspiciados por el derecho económico en Colombia - B. El Marco Analítico de Harvard - C. Bases para la aplicación del gender mainstreaming en los análisis del derecho económico colombiano de cara a los procesos de desarrollo económico - D. Estructura del gender mainstreaming en los análisis del derecho económico en Colombia - III. CONCLUSIONES - Referencias.

Cómo citar este artículo: Hoyos Rojas, L. M. (Diciembre, 2014). El Marco Analítico de Harvard (gender mainstreaming) en los análisis del derecho económico: propuesta para visibilizar a la mujer en los procesos de desarrollo económico en Colombia. Revista de Derecho Público, 33. Universidad de los Andes (Colombia).

El presente documento es parte de la investigación "Derecho económico y género: alianza para la erradicación de los efectos adversos del desarrollo contra la mujer colombiana" y "Víctimas invisibles, conflicto armado y género" original(es) del mismo autor en la Revista de Estudios Latinoamericanos (ReVista Fall, 2013) de Harvard University (Estados Unidos) y en Con-texto. la Revista de Derecho Económico de la Universidad Externado de Colombia (Colombia).

** Abogado de la Universidad del Norte (Colombia). Especialista en derecho constitucional comparado de la Universidad de Chile. Máster (M.Sc.) en estudios latinoamericanos con concentración en derecho, desarrollo económico y género de Houston University (Estados Unidos). Becario para (máster) con concentración en género y desarrollo económico de Harvard University (Estados Unidos). Investigador del grupo de investigación Pensar (en) Género del Instituto de Estudios Sociales y Culturales (Pensar) de la Pontificia Universidad Javeriana de Bogotá D. C. Asesor del Despacho de la directora general del Instituto Nacional para Sordos (Insor), Ministerio de Educación Nacional. Correo: hoyos.luis@javeriana.edu.co 
Introducción

El desarrollo económico incluyente en Colombia empezó a tener rigor con la expedición de la Constitución Política de 1991. En muchas otras partes del mundo, sin embargo, diversas concepciones del progreso y el desarrollo en términos económicos con una representación incluyente venían siendo discutidas desde 1945 (Escobar, 1986, p. 18).

La Constitución del 1991 incluyó un capítulo sobre el régimen económico que, a manera de propuesta transformadora, generó la aparición de principios para la realización del bienestar de todos y todas.

En su sentido más amplio, Colombia definió y modernizó en la reforma constitucional 19901991 el proceso y visión de desarrollo -entendido este en los términos de Kalmanovitz (2001, p. 25), como el "conjunto de factores políticos, culturales y económicos que definen un país como moderno y desarrollado"- que venía adelantándose desde comienzos de los años cincuenta en el gobierno de Mariano Ospina Pérez, con el apoyo de una misión del Banco Mundial (Perry, 1972, pp. 3 y 4), y luego con el Plan General de Desarrollo Económico y Social 19601970, expedido durante la administración del presidente Alberto Lleras Camargo.

De esta manera, la nueva y prometedora Constitución situó en nuestro país lo que se conocía teóricamente como revolución económica (Escobar, 1996, p. 70), dándonos nuevos entendimientos para el proceso de modernización social y económica. Tal visión, no obstante prometedora, siguió refiriéndose a la acumulación de capital para la industrialización -revolución económica propiamente dicha-, sin promover la generación de políticas y análisis para la igualdad y la inclusión social, en el marco de la realización de las capacidades humanas. Situación que ya había sido expuesta en 1958 por Joseph Lebret en su informe Estudio sobre las condiciones del desarrollo en Colombia, donde puso en evidencia que los programas de desarrollo social y económico no correspondían a la realidad nacional por la reiteración del gobierno en generar segregación y exclusión social desde procesos económicos (López, 1994, p. 32).

Cuando en el mundo se hablaba de interacción entre economía y desarrollo desde una perspectiva incluyente o "estrategia de crecimiento y desarrollo con equidad" (Contreras, 2003, p. 22), tal interacción no llegó al país, y los primeros avances que sobre esta se forjaron fueron respaldados por diversas corrientes que no consiguieron instituir alianzas entre enfoques incluyentes y el capital para la generación de oportunidades.

Fue entonces hasta 1997 cuando la Corte Constitucional consiguió establecer un cuerpo axiomático a las promesas inmersas en el desarroIlo, logrando un mandato de optimización que generara la participación activa de todos y todas en los procesos que sufría en aquel entonces el país. La Corte interpretó que los procesos de modernización y de desarrollo en Colombia debían generar el equilibrio razonable entre los beneficiarios de toda la economía, promoviendo 
la realización de oportunidades para todas las personas, más en particular para aquellas de menores ingresos (sentencias C-233 de 1997 y C-150 de 2003).

Con esta posición la Corte dio a comprender que el proceso de desarrollo específico de la reforma constitucional debía contar con formas, mecanismos legales y otra serie de posibilidades que auspiciaran la equidad social y económica. Según su interpretación, era necesario diseñar marcos de análisis social y políticas de inclusión de frente al desarrollo social y económico, que a manera de instrumentos políticos, legales y sociales apuntaran a la generación de indicadores y guías para la equidad (sentencias C-233 de 1997 y C-577 de 2005).

[n]ormas orientadas a establecer la producción, distribución, utilización y consumo de bienes y servicios públicos, buscando la racionalización de la actividad económica con especial énfasis en la protección del empleo y en la promoción de la productividad y la competitividad nacionales. Favoreciendo el cabal cumplimiento de las prerrogativas inherentes a la libre iniciativa y a la vez, procurar la protección del interés público comprometido dentro del marco de las responsabilidades sociales a las que alude la Constitución (sentencia C-233 de 1997).

La Corte marcaría un debate mucho más amplio y antiguo que se refiere a la relación entre derecho, economía y desarrollo social y, a partir de la Constitución de 1991, entre derecho económico e inclusión. Sin embargo, podemos creer que Colombia, en el largo camino hacia el progreso, ha constituido una gran base para la alianza en- tre economía y desarrollo económico con miras a la erradicación de ciertas necesidades sociales. Base que ha evolucionado desde la predominancia de la economía, pues las relaciones entre esta y el derecho han sido marcadas desde la concepción de actividad económica (Currie, 1984, pp. 113-132).

En Colombia solo se vienen considerando como acciones económicas y para el desarrollo económico - entendido en los términos de Del Vecchio (1967, p. 104)-, aquellas que tienen por objeto la adquisición o circulación de bienes y servicios, en las cuales los medios son adecuados a los fines de esta especie. Bajo esta perspectiva, aquellas acciones dirigidas a la adquisición de fines netamente sociales o humanísticos han sido un tanto extrañas a la economía y al desarrollo económico.

Es en la anterior configuración donde el derecho solo tiene un papel subordinado por aqueIlas aproximaciones teórico-económicas que lo instrumentalizan reduciéndolo solo a una competencia de regulación en el marco del desarroIlo. Este enfoque ha generado pocos espacios para la discusión socioeconómica e incluyente, relegada por temas técnico-económicos que se resisten al análisis de ciertas instituciones y conceptos sociales, pero que promueve y asegura la asignación de recursos a través de la participación equitativa en los sectores sociales. El efecto de la aplicación de este enfoque es un mejor desempeño económico que propugna por la eliminación de cualquier forma de discriminación. 
De esta representación nace la importancia de replantear desde un espacio de discusión teórica jurídica un "cómo" que permita la interacción entre economía y desarrollo económico desde una perspectiva social en Colombia. La realidad es que las iniciales aproximaciones teórico-económicas referidas a utilidad, relación medio-fin, actividad y apertura económica, regulación económica y de los mercados, consumidores, competencia comercial, mercado bursátil y de capitales, entre otras, que entendieron en cierto momento histórico el desarrollo y conceptualizaron al derecho como instrumento subordinado por la economía, hicieron pasar al segundo plano la sensibilidad social y el objetivo del progreso que son, en todo momento, las personas (Garay, 1999, p. 33).

Los conceptos económicos con los que se ha entendido el proceso de desarrollo económico en Colombia no han logrado de forma progresiva generar la aparición de sólidos mecanismos de intervención a los sectores menos privilegiados, hoy denominados "sujetos de especial protección constitucional", entendidos en ellos las mujeres, los adultos mayores y las personas con o en situación de discapacidad (Bernat, 2009, p. 32).

Esta es la mayor debilidad que hoy se convierte en una promesa incumplida del desarrollo económico en Colombia, pues en el país históricamente no se han generado coherentes procesos de interseccionalidad entre desarrollo económico, inclusión social y derecho económico, para efectos de incluir aquellas personas en situación de especial protección constitucional, para quienes la aplicación del concepto de desarrollo ha sido incierta (Ocampo, 2000, p. 33).

En el mismo sentido, no hay duda alguna de que las mujeres son, y no han dejado de ser, el sector social que ha padecido históricamente la situación de desventaja en todo este proceso de desarrollo social y económico. Si bien formalmente se reconoce igualdad entre hombres y mujeres, el hecho es que no se puede desconocer que las mujeres han tenido que recorrer un largo camino en el reconocimiento de sus principales garantías sociales, económicas y culturales (Luna, 2003, pp. 16-17), y que aún se justifica la necesidad de una propuesta de análisis del derecho económico con una perspectiva de género que aproxime soluciones a la mujer, contribuyendo a la erradicación de la discriminación que hoy se presenta en Colombia. ${ }^{1}$ De esta forma, este documento tiene como objetivo hacer una propuesta de análisis de la perspectiva de género en el derecho económi$c o$, para demostrar que pese a la existencia de una construcción constitucional, legal y jurisprudencial que responde al desarrollo económico de todos y todas en los sectores socioeconómicos, aún existen temas fundamentales que el derecho económico debe definir para efectos de construir esenciales cambios a favor de la mujer colombiana (Alviar, 2001, p. 22).

\footnotetext{
1 En Colombia las organizaciones feministas se proponen, entre sus objetivos, el "empoderamiento económico" de las mujeres, es decir, el desarrollo en el campo social de sus propias estrategias. Así, buscan trabajar para que los hombres políticos aprendan a compartir su parcela de poder, y fomentar la comunicación entre mujeres políticas (González, 1999).
} 
En definitiva, se partirá de la convicción de que el derecho económico aún tiene mucho que aportar a los procesos de desarrollo económi$\mathrm{co}, \mathrm{y}$ que solamente de un intercambio entre el método analítico del derecho económico y la aplicación del gender mainstreaming o Marco Analítico de Harvard se podrá promover interposiciones jurídico-sociales orientadas a impulsar el bienestar de la mujer colombiana.

De lo anterior nace la importancia de plantear desde la teoría del género en el derecho económico para el desarrollo, una propuesta que prescinda de aquellas teorías y análisis económicos excluyentes generadores del gender blindness o “ceguera de género". Una propuesta que aproxime soluciones a favor de la mujer, contribuyendo a la erradicación de la discriminación que hoy se presenta en Colombia. Una proposición real y social que logre la vinculación de los procesos de desarrollo económico con aquellos sectores menos privilegiados.

Desde esta concepción el género será el cuerpo que dará forma a lo que entenderemos como derecho económico en este documento, para efectos de materializar ideales de equilibrio razonable de desarrollo para el país, inspirados desde la Constitución de 1991.

\section{ASPECTOS HISTÓRICOS DEL DERECHO ECONÓMICO, DEL DESARROLLO ECONÓMICO Y LA PERSPECTIVA DE GÉNERO EN COLOMBIA}

\section{A. Derecho, desarrollo² y mujer en Colombia}

Concebiremos el concepto de desarrollo como aquel avance en los niveles de crecimiento económico, social, cultural y político de una sociedad o país (Stiglitz, 2002, p. 17). Y desde el punto de vista económico, como la generación de oportunidades para la población y los medios para satisfacer sus necesidades.

Para lograr el desarrollo económico debe existir una justa y eficiente distribución de la riqueza nacional, que permita a la población acceder a los servicios básicos (Calabresi, 1978, p. 112). En esta concepción, el desarrollo económico exige para su realización la creación de un entorno en el que las personas puedan ampliar su máximo potencial y llevar adelante una vida productiva y creativa de acuerdo con sus necesidades e intereses (Diez Mintegui, 1996). Si para el desarrollo las personas son la verdadera riqueza, ello implica ampliar las oportunidades para que cada quien pueda vivir una vida que valore (Sen, 1998). El desarrollo económico es

2 Me refiero al desarrollo entendido en la perspectiva social, lo que el derecho económico hace por este, que viene a ser el aspecto más importante, pues se encamina a la atención de aquellas personas que más lo requieren en la sociedad, no tratándose de empresas, industrias o el Estado mismo, sino de las personas en sí y sus necesidades. 
entonces mucho más que el crecimiento económico, que constituye solo un medio - si bien muy importante- para que cada persona tenga más oportunidades.

Para generar más oportunidades lo fundamental es desarrollar las capacidades humanas: la diversidad de cosas que las personas pueden hacer o ser en la vida. Esto es, justipreciar las capacidades sensibles a los procesos de desarrollo económico: haber sido educado, acceder a los recursos necesarios para lograr un nivel de vida digno y poder participar de forma equitativa en el devenir de la comunidad. Sin estas capacidades, se restringe cuantiosamente la variedad de opciones disponibles y muchas oportunidades en la vida permanecen inaccesibles (Sen, 1995, p. 55).

En Colombia, en la última década, la pobreza ha afectado a casi la mitad de la población (PNUD, 2010). Si bien es claro precisar que en el país se adelantan esfuerzos para que los niveles de desigualdad entre sectores de la sociedad no aumenten, ${ }^{3}$ aún se detectan ciertos niveles

3 En septiembre del 2012, el Gobierno Nacional lanzó los Lineamientos de la política pública nacional de equidad de género para las mujeres y el Plan integral para garantizar una vida libre de violencias, constituyéndose en el referente para el diseño y puesta en marcha de acciones sostenibles para la superación de brechas y la transformación cultural, que en el horizonte de los próximos diez años contribuyan al goce efectivo de los derechos de las mujeres en Colombia. El Conpes Social 161 presenta la Política Pública Nacional de Equidad de Género y precisa el plan de acción indicativo para el período 2013-2016, el cual incluye el Plan integral para garantizar una vida libre de violencias. Las problemáticas abordadas y priorizadas en este documento reflejan aspectos centrales de las desigualdades que afectan a las mujeres en nuestro país, evidenciando la pertinencia de su tratamiento intersectorial en forma articulada por parte del Estado. Esto se logrará a través de la puesta en marcha del Plan de Acción Indicativo que precisa objetivos, alcances y acciones de las entidades involucradas, para avanzar en la superación de las brechas de inequidad. de exclusión ${ }^{4}$ que han generado el quebranto constante de oportunidades para que muchas circunstancias de superación persistan como inaccesibles a muchos y muchas (Comité para la Defensa de los Derechos Humanos en Colombia, 2011).

Han sido ciertos grupos poblacionales ubicados bajo la línea de pobreza los que se han visto más afectados por este quebranto constante de oportunidades, que es lo que en últimas ha generado la exclusión: las mujeres, históricamente las menos favorecidas por las dinámicas del mercado, presentan mayores tasas de desempleo e informalidad, con una desventaja relativa en los ingresos laborales. ${ }^{5}$ Sobre todo las mujeres indígenas y afrocolombianas, con discapacidad y en situación de desplazamiento forzado. Debido a su condición de género, etnia, discapacidad o a su situación de desplazamiento, han enfrentado situaciones de carencia de oportunidades e inseguridad social para generar ingresos que les ayuden a superar la situación de vulnerabilidad en la que actualmente

4 En Colombia, las mujeres son definidas como "en riesgo de exclusión social", y en la actualidad son pocos los escenarios donde participan en el ámbito social o público; son las que para subsistir dependen de los recursos sociales, de la manutención por parte de sus familias o parejas; y son quienes no tienen un proyecto de vida propio y aún no se plantean la autonomía, la independencia o la igualdad. El Conpes 161 sobre Equidad de género para las mujeres, aprobado en el 2013, trata de desarrollar una verdadera directriz para que las mujeres en Colombia tengan acceso al proceso de integración social y laboral. Disponible en: https://www.dnp.gov.co/LinkClick.aspx?fileticket=1HWT eFgGXhY\%3D\&tabid=1657

5 Para junio del 2012, la tasa de desocupación para el sexo femenino de Colombia era del 24,2 por ciento, mientras que para los hombres era del 17,1 por ciento y la tasa total era de 20.4 por ciento. El número de hombres ocupados en junio de 2012 para las siete principales áreas metropolitanas fue de 3.206 .000 y el de mujeres de 2.596.000. Lo crítico de todo es que la economía sí generó puestos de trabajo en el tercer trimestre del año, pero no alcanzó a absorber la alta oferta laboral de las mujeres (DANE, 2013). 
se encuentran. ${ }^{6} \mathrm{~A}$ manera de ejemplo la mujer sorda colombiana:

En el país las mujeres sordas no cuentan con los servicios y recursos accesibles a su realidad (Lengua de Señas Colombiana-Lsc), por lo que no les es posible transmitir su situación y necesidades de desarrollo, o simplemente para interponer una denuncia y/o acciones legales. Es válido desde esta representación tomar como punto de partida que en el país coexiste un presunto desconocimiento acompañado de una falta de sensibilidad social por la perspectiva de -mujer y sordera-. Causando para la mujer sorda inaccesibilidad a los servicios de administración de justicia, protección social, campañas de desarrollo. La mujer sorda es un sujeto de derechos que vive procesos de doble discriminación y de la anulación de la persona (aniquilación simbólica), permitiendo exponer al país que los procesos de estigmatización que han vivido históricamente las mujeres con discapacidad son distintos al de cualquier población, tomando como línea base social, que la mujer sorda es más afectada que cualquier otro tipo de mujer con discapacidad, y que la limitación auditiva le restringe ciertas posibilidades educativo-formativas las cuales suponen la adquisición de competencias profesionales y de integración laboral (Hoyos Rojas y Álvarez Castaño, 2014, p. 18).
La existencia de indicadores que señalan que nuestro desarrollo económico se traduce en: estructura económica deficiente, poco ahorro económico por habitante, producción y tecnología desequilibrada, inminente desempleo, diferencias injustificadas en la distribución interna de la riqueza, etc., expone una desigual asignación de los recursos, generando un desaprovechamiento de las oportunidades. ${ }^{7}$ Esto coloca en evidencia que el protagonismo del derecho económico en el desarrollo no ha sido guiado por una política económica socialmente incluyente, ${ }^{8}$ pues la desigual asignación de recursos es producto de escenarios no analizados desde contextos jurídico-económicos, sino desde hegemonías netamente mercantiles (Layne y Shawrs, 1993). Ello pone de manifiesto que no se han perfeccionado en el análisis del derecho económico colombiano instrumentos de política económica incluyente para el desarrollo, como el llamado "enfoque de derechos para el fortalecimiento de las capacidades humanas para el

7 Colombia se caracteriza por una injusta distribución de la riqueza, casi el $50 \%$ vive bajo la línea de pobreza. En primer lugar la historia nos demuestra que los que merecieron el trato igual fueron un pequeño grupo de personas, mientras la gran mayoría: pobres, indígenas, mujeres, niños, etc. fueron simplemente ignorados, jurídicamente inexistentes o no considerados ciudadanos, por tanto no sujetos de derechos (Anónimo, 2011).

8 Más allá de la creación o el fortalecimiento de las instancias encargadas de liderar el desarrollo económico local, para generar un desarrollo armónico y social del país es necesario plantear la implementación transversal de un enfoque incluyente en todos los procesos de desarrollo económico promovidos por los actores locales, con el fin de beneficiar a la población en situación de pobreza y vulnerabilidad. En este esfuerzo es indispensable que haya un liderazgo y coordinación interinstitucional, así como una interlocución con los actores privados y las organizaciones sociales, que facilite intervenciones integrales con eficacia y eficiencia. Esto conducirá a la identificación de estrategias, proyectos e instrumentos que permitan ampliar las oportunidades de inclusión productiva de los grupos en situación de vulnerabilidad en los territorios (Departamento Nacional de Estadística y Departamento Nacional de Planeación, 2010). 
desarrollo" (PNUD, 2006) al que se refirió implícitamente la Corte Constitucional en 1997.

El derecho económico colombiano que en sus análisis ha logrado entender que la realización de las personas solo es posible con la aplicación de un marco para el proceso de desarrollo económico, aún no ha adecuado incluir enfoques de derechos para el fortalecimiento de las capacidades humanas (Bell, 1985, p. 19), que comprende la formulación de nuevos análisis, perspectivas incluyentes y otro tipo de alternativas que buscan la realización de los derechos (OIT, 2008).

Percibir el comportamiento de la economía nacional de frente al desarrollo e igualmente identificar y diferenciar aquellos cambios favorables o desfavorables que se producen en la vida económica al servicio de las personas, ha sido una meta no lograda, lo que significa que la función que ha ejecutado el derecho económico en cuanto a la obligación de proporcionar respuestas eficaces y eficientes no ha sido cumplida. Esta situación hace difícil construir una definición cómoda de desarrollo desde el derecho económico, cuando hoy siguen detectándose tendencias que mancillan el proceso y no han sido corregidas por medio del establecimiento de justas y distribuibles reglas neutrales para todos los actores sociales (Vela, 2005, p. 64).

\section{B. La promesa incumplida y el nuevo paradigma del derecho económico}

Los inicios del desarrollo económico en Colombia señalan un derecho económico subordina- do, concebido solo para efectos de reglamentar la economía, esto es, un derecho sin análisis incluyentes. Un derecho generador de análisis económicos consistentes en estudios y evaluación de las fuerzas y potenciales instituciones del mercado y de la economía (Díaz, 1988, p. 22).

El hecho de que en Colombia se adoptó en el pasado la industrialización por sustitución de importaciones (ISI) como modelo de desarrollo (Amézquita Zárate, 2010, p. 40), generó particularidades que en nuestro presente se convirtieron en un impulso distributivo negativo de la economía para lo social. La ısı fue un modelo de desarrollo que se concentró en lo urbano y en la industria (Clavijo, 2002), lo que hizo que los recursos para lo social fueran inexistentes, además del gran énfasis en la capitalización para la producción de sustitutos y no de formación humana. Lo anterior, acompañado de un derecho económico subordinado por asuntos puramente económicos, financieros e industriales permitió paralizar el impulso distributivo para lo social, que en conjunto con un modelo de desarrollo económico centrado solo en las grandes ciudades, conllevó una injusta y casi inexistente distribución de recursos. Tal contexto logró directamente afectar a la mujer colombiana, la hizo totalmente invisible de todas las relaciones económicas y sociales, pues el Isı al concentrar su capitalización en las urbes y en la industria olvidó que la mujer urbana no era, para finales de la década de los cincuenta, la muestra representativa de aquella población femenina partícipe en todos los procesos de desarrollo (Ballara y Parada, 2009, p. 25). 
En este aspecto de concentrar el capital en las ciudades y las industrias, olvidando las regiones y los distintos contextos de desigualdad en estas, logró un desconocimiento y omisión total de la brecha entre hombres y mujeres respecto al acceso a la propiedad privada, la propiedad de la tierra y al crédito. Lo anterior generó que la mujer colombiana fuera incorpórea en los análisis y las perspectivas incluyentes, pues en Colombia históricamente a la mujer se la aisló socialmente y en lo económico se la relegó mayoritariamente a la actividad doméstica (Torres, 1972, p. 55).

La situación se agravó producto de que bien entrado el siglo XX, las mujeres de Colombia y de algunas partes del mundo tenían restringida su ciudadanía y la mayoría de derechos económicos, sociales y culturales, dado que se las equiparaba a los menores y dementes en la administración de sus bienes, entre otras limitaciones (De Karpf, 1997, p. 48). Este contexto histórico bordó la invisibilidad de la mujer en los análisis del derecho económico, que no lograron señalar que ellas tenían necesidades de concebir un ingreso monetario decente y subsistente como fundamento básico para su manutención y desarrollo como personas.

No se puede dejar de mencionar que el imaginario social y la división de tareas por género/ sexo tampoco fueron asimilados en los análisis del derecho económico, que en sus inicios concibió a la mujer como protagonista responsable del hogar y a los hombres como "ayudantes". Podemos concluir que las mujeres fueron incorpóreas en los análisis del derecho económico, ya que su labor socialmente no se consideró.
Sea como fuera, por conveniencia o no, fue evidente que desde 1920, incluso antes, se vino sometiendo, denigrando y excluyendo a la mujer colombiana de los procesos de desarrollo social y económico (De Melo, 1991, p. 66). La inexistencia de la capacidad jurídica y social para que la mujer pudiera dirigir sus procesos de desarrollo, fue la más paupérrima realidad de una subordinación socioeconómica que para ellas fue un completo atraso histórico (Scott, 1992, p. 88). Es en este mismo momento histórico donde se evidencian los principales efectos de la exclusión de la mujer de los análisis del derecho económico, consolidándose con esto nuevos registros de discriminación por razones de género, los nichos de la llamada violencia basada en género, que para este caso contribuyeron con la deuda del desarrollo y de la economía de la mujer de nuestro país (Borges, 1972, p. 55).

\section{MUJERES REZAGADAS POR LA POBREZA}

La pobreza entre la población femenina es 15 por ciento superior a la de toda la población. De cada 50 horas trabajadas, 30 son sin remuneración.

Aunque Colombia y, en general, los países de América Latina avanzan en las estrategias para la reducción de la pobreza, la situación de las mujeres no mejora al mismo ritmo del promedio total.

Según la Comisión Económica para América Latina (Cepal), el índice de pobreza femenina está por encima del total de cada país. Tal revelación fue hecha durante la XII Conferencia Regional sobre la Mujer, que se instaló ayer en República Dominicana y se extenderá hasta el viernes.

Las mujeres son mayoría, el 50,9 por ciento de la población latinoamericana, y ha habido un notorio incremento de mano de obra femenina en el mercado laboral, ellas quedan rezagadas frente a los avances contra la pobreza. En todos los países del continente "la tasa de pobreza de las mujeres de 20 a 59 años de edad excede en un 30 por ciento o más a la de los hombres de edad similar", anota el informe de la Cepal. 
La Cepal estima que, si bien en las últimas décadas ha habido un mayor ingreso de mujeres al mercado laboral, no parecen estar entrando a actividades que les permitan tener una mejor calidad de vida. "El tipo de empleo que tienen las mujeres está más relacionado con labores de cuidado (como los ámbitos de salud, educación y cuestiones sociales en general) y se desempeñan en menor medida en ámbitos relacionados, por ejemplo, con las tecnologías".

En Colombia, si la pobreza asciende al 32,7 por ciento, entre las mujeres es de 37,6 por ciento.

Fuente: http://www.portafolio.co/finanzas-personales/ mujeres-se-rezagan-avances-pobreza, octubre 16/ 2013.

Ahora bien, para generar en Colombia cambios respecto al protagonismo de la mujer en los procesos de desarrollo, resulta del todo interesante asumir una nueva concepción del derecho económico, una que sea capaz de aminorar impactos de exclusión. Es necesario iniciar esbozando que el derecho económico debe ser comprendido como un conjunto de principios y normas sustancialmente de derecho constitucional, que cumple finalidades sociales y no en el antiguo modelo que admitió históricamente la exclusión sociofemenina (Cuadra, 1990, p. 77).

La Constitución de 1991 revivió en Colombia la doctrina respecto al derecho constitucional económico, donde muchos autores apuntan que nació constitucionalizado con un enfoque netamente incluyente, desde la perspectiva de los derechos humanos y del goce efectivo de los derechos económicos y sociales (Calderón, 2011, p. 33). Esta aproximación doctrinal fue desarrollada por el profesor Kresalja Rosello (2004), quien acordó que el efectivo derecho económico era "el conjunto de principios y normas jurídicas que organizan la economía de un país y facultan a la autoridad para regularla en armonía con los valores de la sociedad nacional formulados en la Constitución".

Kresalja Rosello dio a comprender que el derecho económico no se agotaba, ni mucho menos, en la Constitución y la economía, sino que comprendía el conjunto de principios, normas y medidas jurídicas en sus muy diversas jerarquías y especies, que consentían la realización social de todos. Visión que para todos los efectos es tomada como válida para esta investigación y que es en todo momento ajustable en Colombia.

Hay que instruir en la asimilación de que el derecho económico tiene una naturaleza científico-social siempre que se la examine con una metodología interdisciplinaria e interseccional no propia de la pura y estricta teoría económica (Posner, 1993, p. 22). Se asimila así al derecho económico en una visión y paradigma jurídicoincluyente, que de ser aplicada no hubiera excluido a las mujeres. El paradigma está integrado por categorías jurídicas (elemento formal) y socioeconómicas (elemento material), de manera que su objeto de estudio pueda ser bicéfalo e interdisciplinario, para lograr encontrar los enlaces para la distribución de los recursos en materia económica y social.

Pero, ¿cómo se reafirmaría el derecho económico en la realidad colombiana para efectos de aminorar el impacto adverso ocasionado a la mujer en los procesos de desarrollo económico?

Las primeras aproximaciones fueron desarrolladas en los Informes sobre Desarrollo del Progra- 
ma de las Naciones Unidas para el Desarrollo (PNUD), donde proponen el análisis de los valores y formas de ejercicio del poder, de las élites y de la ciudadanía, enfatizando las experiencias positivas y sus resultados en favor del desarroIlo incluyente, buscando establecer el bienestar humano como objetivo central del desarrollo mediante la transformación de las relaciones de género fuera de una visión economicista (PNUD, 2005).

En este entendido, desde el derecho económico debe plasmarse que la visión de la economía para el desarrollo debe ser real, social e incluyente, y como tal debe ponerse en ella radical atención, de tal manera que las decisiones y actividades institucionales que se realicen en esta materia respondan a una visión incluyente de la cuestión.

Es necesario desde el derecho económico proponer la igualdad o la reducción de las desigualdades o asimetrías, como elemento sine qua non del desarrollo, modificando la estructura económica de producción y distribución para que se produzca un crecimiento equitativo, exigiendo también programas de desarrollo e inversiones que no dependan únicamente de los mecanismos de mercado, sino de los procesos de cooperación con enfoque incluyente (Witker, 1978, p. 72), por lo que es meritorio fomentar el reparto de la participación plena e igualitaria en la toma de decisiones de hombres y mujeres. De ahí que el objetivo del desarrollo en Colombia sea alcanzar una igualdad como apunta la Constitución de 1991, tanto en el acceso como en el control de los recursos económicos, y esto es a lo que debe responder el derecho económico.

\section{EL MARCO ANALÍTICO DE HARVARD (GENDER MAINSTREAMING) EN LOS ANÁLISIS DEL DERECHO ECONÓMICO EN COLOMBIA}

\section{A. Necesidad de la teoría y perspectiva de género en los procesos de desarrollo económico auspiciados por el derecho económico en Colombia}

La igualdad de género es considerada de suma importancia con el fin de lograr el bienestar para todas las mujeres y hombres, y de llevar a cabo cambios sociales e institucionales que produzcan un desarrollo con equidad y crecimiento. La igualdad de género se refiere a la igualdad de derechos, responsabilidades y oportunidades que deberían beneficiar a todas las personas, sin importar si son hombres o mujeres (oIt, 2008).

El hecho de que la mayor parte de la propiedad privada esté en manos de los hombres (González, 2008, p. 57) tiene consecuencias sociales de largo alcance y profundas repercusiones en la estructura misma de la sociedad que, entre otros, resultan en la feminización de la pobreza. La falta de derechos iguales al acceso y propiedad de la tierra y a la herencia tiene como consecuencia el empobrecimiento y la inseguridad de las familias. La perspectiva de género, además, permite distinguir entre los diferentes aportes de mujeres y hombres a la sociedad desde el punto de vista económico de cara al desarrollo, 
con el propósito de no dejar la propiedad y los recursos en un solo género, sino distribuirlos apropiadamente (Cortina, 2003, p. 101).

La perspectiva de género en el derecho económico permitiría concebir la participación de todos para la realización social de los derechos constitucionales económicos, con el fin máximo al que aspira la Carta en el continuo perfeccionamiento del concepto de Constitución económica. Un primer avance de la alianza derecho económico y género aplicado alcanzaría a generar un coherente análisis de las necesidades, para efectos de guiar la asignación de los beneficios y las cargas de la actividad económica de forma objetiva. La categoría analítica del género dotaría al derecho económico de una nueva fuente que propugnaría por el encuadramiento de los fenómenos sociales de los más vulnerables a la norma y realidad económica, bajo los principios de equidad, igualdad y proporcionalidad (Cortina, 2003, p. 101). Con ello se busca un desarrollo incluyente que logre concertar la capacidad humana y la reciprocidad entre hombres y mujeres, como principio de la prosperidad y el bienestar socioeconómico.

La alianza derecho económico y género representaría erigir una sociedad socioeconómica que garantice la total inclusión de mujeres y hombres en la dinámica del desarrollo de estrategias específicas, para que las personas o los grupos de la población que se encuentran en condiciones precarias puedan mejorar su situación actual, superando la clásica visión del derecho económico.
Por ello, introducir de manera transversal o eje conductor el género, como categoría de análisis y como método de identificación y corrección de desigualdades en el derecho económico, implica a futuro condicionamientos a las políticas públicas existentes en Colombia para la equidad y a los planes, programas, proyectos y mecanismos de trabajo públicos en el contexto de oportunidades sociales y de desarrollo en nuestro país (Alta Consejería para la Equidad de la Mujer, 2012 y Conpes Social 161). Son necesarios marcos de análisis que, como el de género, ofrecen una comprensión más amplia del conjunto de la dinámica económica y de las distintas actividades que el método de estudio del derecho económico no prevé ni previó jamás en Colombia (Hoyos, 2013). En definitiva, el derecho económico con la perspectiva de género estaría orientado a:

1. Propender por el desarrollo objetivo de la igualdad y la no discriminación arbitraria que haga el Estado y sus organismos en materia económica. En Colombia persiste el apotegma que predica: "fuera del ámbito económico para el derecho no hay más horizontes". En efecto, la meta de la perspectiva de género en el derecho económico colombiano delinearía una representación diferente sobre el concepto actual de regulación económica del Estado. Actualmente lo que ocurre es que el objeto de estudio del derecho económico hace énfasis en los análisis económicos y no en las diferencias sociales ni en las repercusiones-contradicciones que ello tiene para la propia economía y para la sustentabilidad humana; no considera las asimetrías sociales y económicas.

2. Propender por el desarrollo de una coherente remuneración para todos sin importar la ac- 
tividad económica, garantizando el respeto a la moral y al reconocimiento de las capacidades humanas de todos y todas. Un ejemplo claro de la inexistencia del enfoque de género en la aplicación del derecho económico en Colombia son las llamadas "brechas salariales por género", las cuales son profundas y persistentes.

Todos los estudios que se han referido al tema han encontrado que los hombres reciben una remuneración mayor que las mujeres, a pesar de que se documenta que las mujeres han aumentado su participación en el mercado laboral $\mathrm{y}$, en promedio, tienen más años de estudios que los hombres. No obstante, lo que sorprende es que las mujeres tienen, en promedio, 6\% más años de educación que los hombres en el grupo de asalariados, por lo cual es poco probable que la explicación esté fundamentada en este hecho y posiblemente sí exista algún grado de discriminación por género en el mercado laboral colombiano. Con la incorporación de relaciones equitativas en género serias, se superarían estas realidades injustificadas presentes en la economía (Trubek y Dezalay, 1994), y la aplicación del principio de equidad social se convertiría en el indicador de realización social entre hombres-mujeres.

En definitiva, la propuesta de la inclusión de género en las relaciones del derecho económico instauraría la modificación de los fundamentos de la política económica, para contribuir a la erradicación de los efectos adversos del sector económico hacia los involucrados, concretamente hacia la mujer.

\section{B. El Marco Analítico de Harvard}

Desde 1995, cuando la IV Conferencia Mundial de Mujeres de Beijing definió en su plataforma de acción el gender mainstreaming como estrategia central, las mujeres políticas y los círculos comprometidos con la igualdad de género comenzaron a alimentar esperanzas de lograr, a partir de este nuevo enfoque, mejores resultados que los obtenidos hasta ese momento. De hecho, la estrategia apunta a obtener mayor impacto en el objetivo de superar las discriminaciones sociales de género y alcanzar mayores niveles de igualdad. ${ }^{9}$

El mainstreaming de género, traducido al español como "transversalidad", puede ser entendido en variados sentidos: como una estrategia que involucra a todos los actores sociales en la búsqueda de la igualdad de género, o como la denominación de determinadas herramientas para el análisis de género. En realidad, se trata de un enfoque transformador, dotado de un cuerpo teórico propio, que apunta a analizar los impactos diferenciados del sistema de género en varones y mujeres, y permite tener en cuenta las especificidades de las personas como seres integrales y, al mismo tiempo, ayuda a implementar medidas para corregir las desigualdades (Frey, 2003b, p. 112).

En las primeras iniciativas de la segunda mitad de los ochenta, la aplicación del enfoque de

$9 \quad$ Ver al respecto: Berlín Dietz y Regina Frey. (2003). Gender im Mainstreaming. Königstein im Taunus: Urike Helmer Verlag. También Emilie Hafner-Burty y Mark Pollack. (2002). Mainstreaming Gender in Global Governance, European Journal of International Relations, 8 (3), 339-373. 
género a proyectos centrados principalmente en poblaciones de bajos ingresos, en comunidades de base, especialmente en países del Tercer Mundo, con claro propósito de favorecer a las poblaciones femeninas de esos estratos, fue uno de los cometidos desarrollados por la comunidad internacional. Los paradigmas que en este sentido dominaron el escenario fueron básicamente los creados por Caroline Moser a partir de sus sistematizaciones conceptuales, los de Kate Young y Karen Levy y los del Marco Analítico de Harvard.

Gracias a estos esfuerzos se consolidó un marco de herramientas conceptuales que ha perdurado como una importante base analítica e interpretativa para los estudios de género no previstos en los marcos referenciales del desarrollo.

Desde esta perspectiva, el gender mainstreaming nació a partir del enfoque denominado gender and development (GAD), en español "género y desarrollo", que se fundamenta en el Marco Analítico de la Universidad de Harvard, uno de los primeros modelos de análisis creados en función de género (Overholt, Anderson, Cloud y Austin, 1988, p. 106).

En sus inicios, el enfoque GAD apuntó a sacar a las mujeres de sus lugares de aislamiento y a dar visibilidad a las estructuras que hacen posible la inequidad de género. Sus herramientas fundamentales fueron, en primer lugar, los entrenamientos en género (gender training) con el doble objetivo de sensibilizar, por una parte, y capacitar, por otra. Algo completamente fundamental para lo que hoy denominamos los análisis de género.
Pero, con la incorporación del concepto de género desde el GAD, se constituyó sin mencionarlo explícitamente un primer intento de incorporar la perspectiva de género al mainstream de las políticas y las instituciones, lo que luego evolucionaría hacía el gender mainstreaming en la década de los ochenta (Moser, 1988, p. 106).

Desde esta perspectiva, el gender mainstreaming, que no representa hasta el momento una propuesta acabada, y cuya denominación incluso suele ser objeto de discusión y planteamiento de nuevas alternativas -tales como: "mainstreaming de género", "transversalización de género", "transversalización del enfoque de género", "enfoque integrado de género", "adopción de criterios de género" e incluso "enfoque de género y empoderamiento"-, logra ligar los procesos de desarrollo adelantados desde el derecho económico a políticas públicas incluyentes.

Fue así como, adoptado primero por las instituciones que promovían políticas para el desarroIlo, el gender mainstreaming comenzó a proyectarse en la arena macropolítica a partir de la IV Conferencia Mundial de la Mujer, de Beijing. La Plataforma de Acción de Beijing pone en claro la existencia de sistemas de género en las diferentes sociedades, el funcionamiento desigual de estos y las distintas dimensiones de los problemas y desigualdades de género que son resultado de este carácter sistémico: simbólica, normativa, subjetiva y relacional. Se reconoce que ningún campo es neutral al género y que es necesario profundizar el análisis multicausal y la integralidad de la acción, y que el Estado es el responsable de hacerlo. 
En este contexto, se rejerarquiza el rol de las oficinas de la mujer, que se conciben como instancias de coordinación de políticas, responsables de liderar el proceso de gender mainstreaming e influir ya no solo en las políticas específicamente orientadas a la mujer, sino en el conjunto de las políticas públicas (PNUD, 2005).

Así entonces, el mainstreaming tiene como propósito integrar el interés en la igualdad de géneros en todas las políticas, programas, procedimientos administrativos y financieros y en el marco cultural de la institución u organización. Se logra entonces definir como el proceso de evaluar las implicaciones que tiene para hombres y mujeres cualquier acción que se planifique, incluyendo las de tipo legislativo, las políticas o los programas en todas las áreas y a todos los niveles de desarrollo. Es una estrategia para hacer de las experiencias y necesidades o intereses de hombres y mujeres una dimensión integral en el diseño, implementación, monitoreo y evaluación de las políticas y los programas en todas las esferas políticas, sociales y económicas, a fin de que hombres y mujeres se beneficien por igual y desaparezca la desigualdad. El objetivo final es lograr la igualdad de géneros (Strassman, 1999), puesto que:

1. El gender mainstreaming es un proceso estratégico. Es una estrategia, no es un fin en sí mismo, el fin es la igualdad; con lo cual podemos entender que de cierta manera representa una opción, un instrumento estratégico para alcanzar la igualdad. De esta primera aproximación podemos deducir, elementalmente, que el empleo del gender mainstreaming exige el uso de herramientas estratégicas. Esto que puede parecer una simpleza, no lo es de cara a algunas experiencias concretas observables en esfuerzos nacionales o locales de algunos países que se proponen, por ejemplo, planes de igualdad bajo la denominación de mainstreaming de género, y para su diseño solo emplean herramientas normativas, que no consideran los diagnósticos estratégicos que habría que desarrollar para varias tareas y donde el plan mismo carece de esta condición.

2. El gender mainstreaming tiene como propósito el logro de la igualdad de mujeres y hombres, con lo cual hay un tácito reconocimiento de que la desigualdad es un problema público. Supone considerar la eliminación de las desigualdades de género y el establecimiento de la igualdad como dimensión o referencia indispensable en todas las fases del proceso de políticas públicas (y aún en el caso de las políticas de las organizaciones privadas que promueven políticas de igualdad), así como en el orden institucional y organizacional que les da soporte, en su ejecución y evaluación. Este es el sentido del término en inglés mainstreaming, que aproximadamente significa en español "la corriente principal", y cuyo alcance, en este caso, creemos que se aproxima a la idea de que el principio de igualdad impregne como referencia sustantiva las decisiones de política y su implementación y evaluación. Este último aspecto se torna vital dado el hecho de que, como ambas definiciones expresan, es de la mayor importancia monitorear los impactos diferenciales que las decisiones tienen en mujeres y hombres.

3. Su aplicación abarca todos los órdenes y niveles de la gestión institucional u organizacional: leyes y todo otro mandato de políticas, programas y proyectos en todas las áreas y niveles sociales, políticos, culturales y económicos. 
4. Debe ser asumido en todos los aspectos o fases operativas de la gestión o proceso de las políticas: diseño, implementación, monitoreo y evaluación.

5. Sus criterios metodológicos fundamentales son:

a. integrar globalmente las experiencias, los intereses y las necesidades de las mujeres y los hombres en todas las iniciativas de cualquier tipo y alcance;

b. evaluar las implicaciones que tenga cualquier acción realizada en mujeres y hombres;

c. garantizar beneficio igual para hombres y mujeres.

6. Supone contar con el apoyo de un equipo calificado a cargo de la dirección, coordinación y apoyo a la ejecución de las tareas correspondientes.

Estos supuestos esenciales constituyen el corazón mismo del gender mainstreaming y alrededor de ellos se ha venido construyendo el conjunto de exigencias y operaciones que demandan su real cumplimiento. No se trata entonces de una acción o programa o estrategia singular, sino, como su nombre indica, una presencia integrada e integral en todas las decisiones y acciones de las organizaciones inmersas en el desarrollo. Más específicamente es una estrategia para asegurar que:

1) La igualdad de hombres y mujeres esté incluida en todas las fases menores o mayores del proceso de toma de decisiones de una organización y desarrollo y,
2) Que el producto o resultado de las decisiones que se hayan tomado sea sistemáticamente monitoreado en lo que se refiere a su impacto en la igualdad de géneros. Por lo que, una iniciativa efectiva de género en el mainstream requiere la interacción compleja de numerosas habilidades y competencias usualmente coordinadas en un equipo de trabajo integrado para tal fin.

\section{Bases para la aplicación del gender mainstreaming en los análisis del derecho económico colombiano de cara a los procesos de desarrollo económico}

En Colombia, la Oficina del PNUD logró situar la Ilamada “Estrategia de Género 2007-2008” con el objetivo de institucionalizar e integrar el género en todos sus ámbitos de trabajo (PNUD, 2005).

La estrategia incorporó los principales mandatos y acuerdos internacionales en materia de desarrollo y género, y los mandatos del Consejo Económico y Social relativos a la inclusión del enfoque de género en todas las políticas y programas del Sistema de Naciones Unidas. En un primer intento la oficina en Colombia adaptó el concepto de TG -transversalidad de género- del denominado Guidance Note on Gender Mainstreaming and Social Exclusion in Research (DFI Research, 2009).

Así, aprobado por la Junta Directiva del PNUD Colombia en 1997, se asume la transversalización de género como una transformación profunda de la organización en todos los niveles. Sin embargo, dada la actual situación de rede- 
finición de la estructura interna de la Oficina en Colombia, se consideró viable comenzar el proceso de TG focalizando los esfuerzos en el Área de Programas.

Por ello, la estrategia en Colombia solo se centró fundamentalmente en el fortalecimiento de las capacidades técnicas para la TG del personal del Área de Programas y en la identificación y priorización de acciones positivas y de género al interior de los programas ya en marcha o en proceso de definición.

Por lo anterior es significativo señalar que tal propuesta no fue concebida como una posibilidad en Colombia que aprobara incorporar acciones tendientes a promover un cambio económico y político a favor de relaciones de género más equitativas en los procesos de desarrollo nacional desde los análisis económicos del desarrollo formulado y plasmado en políticas. Más bien fue implementado como un programa piloto de sensibilización a funcionarios y programas internos que sobre la equidad de género tuvieran algún tipo de impacto sectorial. Si bien en Colombia es clara la existencia de un primitivo avance para la aplicación del gender mainstreaming, es importante fijar que no hay certeza política y científica sobre su aplicación en los análisis del derecho y la economía en lo que concierne.

El gender mainstreaming se propone en los análisis del derecho económico como un proceso de cambio que demanda una perspectiva estratégica capaz de replantear las dimensiones sociales que podrían o convendrían caer bajo la influencia de este cambio; pero idénticamente, las dimensiones institucionales de las políticas y los procesos de desarrollo que la ponen en marcha, y las implicaciones que en otros órdenes sociales como los de raza, edad, etnia, clase y otros deberían ser razonados colectivamente, así como los patrones de igualdad dominantes que aparecen dentro del sistema colombiano de las relaciones de poder entre los géneros (Hoyos, 2012). Análisis que efectivamente no existe en Colombia.

Esto significa que la estrategia exigiría desde el derecho económico, para un cambio como el que supone, liquidar las desigualdades de género, una perspectiva progresiva de mediano o largo plazo, donde se asuman las necesarias factibilidades políticas del desarrollo que no dependen solo de la preexistencia de voluntad política sino de los procesos de legitimación de las iniciativas (Gálvez, 2001), para evitar que la propuesta se convierta en un anuncio de "guerra entre los sexos", como a menudo enuncian quienes se resisten desde sus actitudes a la equidad de género en el desarrollo.

Se propone igualmente que desde los análisis del derecho económico aplicados al desarrollo, se haga un trabajo estratégico con las condiciones del contexto social donde puedan aparecer las reales y efectivas oportunidades, lo que le exigirá al derecho económico la necesidad de trabajar claramente con propuestas y previsiones de empoderamiento fuera del marco de los actuales análisis de la pura teoría económica (Roldán García, 1988, p. 112). Se cumpliría así de forma indefectible con los postulados de distribución, asignación y equidad que establece la 
norma constitucional en el artículo 334 para los procesos de desarrollo en nuestro país.

El gender mainstreaming suprimiría la invisibilidad de la mujer en los análisis del derecho económico y de la economía en Colombia, porque instalaría desde los análisis una visión de desarrollo complementado con las acciones de integración de la mujer a este. Igualmente, generaría la integración de la mujer a la producción en condiciones de igualdad en cuanto a oportunidades y estímulos, pues ella sería un referente obligatorio de análisis económico de la sociedad colombiana. Ello reforzaría todas aquellas instancias organizativas de mujeres que les permitan tomar conciencia de su subordinación de género, clase, etnia, etcétera, como fuerza política desde la economía y el desarrollo.

\section{Estructura del gender mainstreaming en los análisis del derecho económico en Colombia}

La aplicación del gender mainstreaming como enfoque propuesto a los análisis del derecho económico en Colombia deberá considerar los tres componentes fundamentales que implica instalarlo en el análisis de los procesos de desarrollo de un país. Estos son: 1) el contexto, 2) la aplicación del proceso de análisis de la asimetría de género en el desarrollo y 3) las estructuras y mecanismos.

\section{- El contexto de inequidad}

Es necesario incorporar a la estructura del gender mainstreaming el contexto de inequidad aplicado ya en Colombia (Gómez, 2003, p. 203), pues en el desarrollo de muchos años nos ha permitido conocer la situación socioeconómica del país. Esta etapa posibilitaría dimensionar los datos desagregados por sexos en la participación laboral, desempleo, participación social y política, educación, salud, violencia de género, emigración e índice de desarrollo humano.

Desde el derecho económico en Colombia, se efectuaría bajo un análisis que demuestre una real prosperidad o un real fracaso, no solo en términos de la factibilidad política del desarroIlo, sino en términos de las bases jurídicas o normativas adecuadas que acuerdan el bienestar fáctico de la mujer. Es decir, que el derecho económico en esta faceta deberá analizar si en Colombia hay mujeres incluidas en la toma de decisiones y con un soporte financiero digno, considerando la incidencia problemática de las desigualdades de género en la situación general de la sociedad, que a ellas les afecta en todos los grados existentes en el país.

- La aplicación del proceso de análisis de las asimetrías de género en el desarrollo

Hace referencia al análisis que permite conocer los complejos procesos para explicar cómo se están estructurando y expresando los ámbitos de lo femenino y lo masculino y cuáles son los símbolos y características que los definen y representan como construcciones culturales opuestas y simétricas en el proceso de desarroIlo. Esta fase del gender mainstreaming coloca en evidencia las relaciones simétricas o asimétricas entre hombres y mujeres en el desarrollo, 
el rol social asignado a cada sexo y la adquisición e identidad genérica que mejora o empeora la realización de los sexos en los procesos de desarrollo social y económico.

\section{- Las estructuras y los mecanismos}

Hacen referencia a la existencia institucional de entidades encargadas de la aplicación de programas de reconstrucción para la equidad en el desarrollo, desde una perspectiva de género que permite reconocer no solo las necesidades de las personas sino también sus estrategias y sus acciones para ayudarse a sí mismas. Se contribuye así a reconocer la existencia de relaciones desiguales entre hombres y mujeres, lo que constituye la base para conocer las diferentes necesidades y capacidades de ambos, especialmente para la mujer.

En Colombia las estructuras harán referencia a las instituciones responsables de la estrategia, que deben tener las condiciones básicas de ser desconcentradas en puntos focales articulados al organismo que liderará el proceso, el cual deberá contar a su vez con un equipo con capacidades para conducirlo y gerenciarlo. Todas ellas deberían seguir el análisis revelado por el diseño del plan desde la aplicación del proceso de análisis de las asimetrías de género en el desarrollo. Supremamente los mecanismos serán los instrumentos variados que utilizará en todo momento el derecho económico para generar los impactos de género sobre los procesos de desarrollo, los cuales serán: a. el constante análisis de género,

b. la producción y procesamiento de información,

c. la concientización y capacitación, y

d. el seguimiento y evaluación.

A esto se le conocería como un verdadero "análisis del derecho económico con un enfoque basado en género" (Grupo Faro, 2007) caracterizado principalmente por el estudio de las políticas y de las instituciones, tanto desde la perspectiva positiva (de la explicación y predicción de relaciones de causa y efecto de lo existente) como en la normativa (del deber ser, más allá de la explicación y la predicción, utilizando juicios de valor y distribución equitativa), que logren racionalizar la economía con el fin de conseguir el mejoramiento de la calidad de vida de todos y todas. Con esto se provocaría la participación femenina en el proceso de desarrollo del país y la economía. La mujer colombiana lograría reivindicarse en posiciones directivas en las empresas y en el gobierno, lo que indudablemente y de forma natural generaría un bienestar fructífero para ellas y para todos.

\section{CONCLUSIONES}

1. El derecho económico coordinado con el desarrollo del país debe responder a la exigencia de valorar las capacidades y competencias humanas que establece la Constitución Política, tomando como punto de partida el principio de participación equitativa que ava- 
la la realización social de los derechos constitucionales y económicos de todas y todos.

2. La perspectiva de género inmersa en las relaciones del derecho económico favorecería a la mujer colombiana, en razón a que nuestra sociedad sigue fallándole en momentos claves de su vida, particularmente en su realización socioeconómica, principales daños hechos en su adolescencia y vejez, pese a que se han realizado algunos progresos desde el derecho y la economía auspiciados por la Constitución Política.

3. La alianza entre derecho económico y la aplicación del gender mainstreaming, como mecanismo para hacer realidad la erradicación de la invisibilidad de la mujer en los procesos de desarrollo en Colombia, denotaría principales cambios en las relaciones laborales y en las condiciones de trabajo surgidos a raíz de los programas de ajuste estructural y las transformaciones ocurridas como consecuencia del enfoque en la economía del país. Se reivindicaría la obligatoriedad de los conceptos de participación y distribución equitativa en los sectores activos de la economía, como muestra eficaz de acciones reales que fijen la aparición de un enfoque en la asignación de roles sin discriminar competencias humanas.

4. La propuesta de los análisis del derecho económico a los procesos de desarrollo social y económico del país con un enfoque basado en género, caracterizaría principalmente un cambio en el estudio de las políticas públicas y de las instituciones en el marco del actual Estado constitucional, modificando la perspectiva positiva de la explicación y predicción de relaciones de causa y efecto de lo existente respecto al empoderamiento de la mujer, como en la normativa del deber ser, más allá de la explicación y la predicción, utilizando juicios de valor y distribución equitativa que logren racionalizar la economía con el fin de conseguir el mejoramiento de la calidad de vida de la mujer colombiana.

5. Finalmente, los análisis del derecho económico de cara a los procesos de desarrollo social y económico del país, con un enfoque basado en la perspectiva de género y en la aplicación del gender mainstreaming, permitirían establecer los fundamentos de un modelo económico y social de desarrollo y equidad, con énfasis en la brecha entre los aportes que las mujeres realizan a las economías del país y los beneficios que reciben.

\section{Referencias}

Alta Consejería Presidencial para la Equidad de la Mujer. (2012). Lineamientos de la política pública nacional de equidad de género para las mujeres 2012-2022. Bogotá: Presidencia de la República de Colombia. Prosperidad para todos.

Alviar, H. (2001). Leon Duguit and the 1936 CoIombian Constitutional Reform. Harvard Law School, (22), 177-188. 
Amézquita Zárate, P. (2010). La política de industrialización por sustitución de importaciones. El contexto colombiano. GCG Georgetown Univerversity-Universia, 4(2), 4-53.

Anónimo. (2011). Informe para el tratamiento a las presas politicas en Colombia. Comité para la Defensa de los Derechos Humanos de CoIombia.

Ballara, M. y Parada, S. (2009). El empleo de las mujeres rurales, lo que dicen las cifras. New York: FAO-CEPAL.

Bell, D. (1976). El advenimiento de la sociedad postindustrial: un intento de prognosis social. Madrid: Alianza Editorial.

Bernat, L. (2009). Análisis de género de las diferencias salariales en las siete principales áreas metropolitanas colombianas: ¿evidencias de discriminación? En Consejería Presidencial para la Dignidad de la Mujer, Cuadernos PNUD (págs. 65-144). Bogotá: PNUD, Colombia.

Borges, A. (1972). La mujer-pobladora en los orígenes americanos. Sevilla: Escuela de Estudios Hispano-Americanos.

Calabresi, G. (1972). The Cost of Accidents: A Legal and Economics Analysis. New Haven, Conn.: Yale University Press.

Calderón, J. J. (2011). La constitucionalización del derecho privado. La verdadera historia del impacto constitucional en Colombia. Bogotá:
Universidad de los Andes, Universidad del Rosario y Editorial Temis.

Clavijo, S. (2002). Reflexiones sobre política monetaria e inflación objetivo en Colombia. Borradores de Economía, 141.

Comité para la Defensa de los Derechos Humanos de Colombia. (2011). Informe para el tratamiento a las presas políticas en Colombia. Bogotá: Comité.

Contreras, D. (2003). Poverty and inequality in a rapid growth economy: Chile 1990-1996. Journal of Development Studies, XXXIX(3), 181-200.

Corte Constitucional de Colombia. Sentencia C-150 del 25 de febrero de 2003. M. P.: Manuel José Cepeda Espinosa.

Corte Constitucional de Colombia. Sentencia C-233 del 15 de mayo de 1997. M. P.: Fabio Morón Diaz.

Corte Constitucional de Colombia. Sentencia C-577 del 25 de julio de 2006. M. P.: Humberto Antonio Sierra Porto.

Cortina, A. (2003). Género en la cooperación al desarrollo: una mirada a la desigualdad. Madrid: Acsur-Las Segovias.

Cuadra, H. (1990). Reflexiones sobre derecho económico. En H. Cuadra y M. Kaplan Estudios de derecho económico (Vol. I). México D. F.: Universidad Nacional Autónoma de México. 
Currie, L. (1984). Evaluación de la asesoría económica a los países en desarrollo: el caso coIombiano. Bogotá: Fondo Editorial Cerec.

De Karpf, A. (1997). Balance de 40 años del voto femenino. En 40 años del voto de la mujer en Colombia. Cali: Gobernación del Valle del Cauca. Bogotá: Dirección Nacional de Equidad para las Mujeres.

De Melo, J. (1991). Fiscal Issues in Adjustment: an Introduction. California: University Press and World Bank.

Del Vecchio, G. (1967). La teoría económica del crédito. Centenario della Nascita. Milán: F. Angeli.

Departamento Nacional de Estadística y Departamento Nacional de Planeación. (2002). Misión para el empalme de las series de empleo, pobreza y desigualdad. Bogotá: DNP y DANE.

Departamento Nacional de Estadística. (2013). Informe del desempleo por sexo en Colombia, 2012-2013. Bogotá: DANE.

Departamento Nacional de Planeación. (2013). Primer informe de seguimiento Conpes Social 161: equidad de género para las mujeres. Bogotá D.C.: DNP y ACPEM.

Department for International Development DFID Research. (2009). Guidance Note on Gender Mainstreaming and Social Exclusion in Research. Recuperado de https://www. abdn.ac.uk/sustainable-international-develo-
pment/documents/GuidanceNote_Gender_ SE.pdf

Dezalay, Y. y Trubek, D. (1994). La restructuración global y el derecho: internacionalización de los campos jurídicos y la creación de espacios transnacionales. Pensamiento Jurídico, 1-40.

Díaz, L. (1988). El derecho económico y la integración de América Latina. Bogotá: Editorial Temis S. A.

Díez Mintegui, C. (1996). Deporte y construcción de las relaciones de género. Gazeta de Antropología, (12), 93-100.

Escobar, A. (1986). La invención del desarrollo en Colombia. Lecturas de Economía, (20), 9-35.

Escobar, A. (1996). La invención del Tercer Mundo: construcción y deconstrucción del desarrollo. Bogotá: Grupo Editorial Norma.

Faini, R. y De Melo, J. (1991). Fiscal Issues in Adjustment: an Introduction. Washington D. C.: World Bank, Policy, Research, and External Affairs.

Frey, R. (2003). Gender im Mainstreaming, Geschlechtertheorie und -praxis im internationaIen Diskurs. Königstein: Ulrike Helmer Verlag.

Gálvez, T. (2001). Aspectos económicos de la equidad de género. En Serie Mujer y Desarrollo. Santiago de Chile: Cepal, Eclac, Unidad Mujer y Desarrollo. 
Garay, L. J. (1999). Construcción de una nueva sociedad. Bogotá: Tercer Mundo Editores.

García, E. (2004). Género, políticas locales e intervención social: un análisis de los servicios de bienestar social municipal para la población femenina en España. Madrid: Editorial Complutense.

Gender in Development Programme. Learning and Information Pack. N. Y. (2010). Obtenido de United Nations Development Programme: http://www.undp.org/content/dam/undp/library/gender/Institutional\%20Development/ TLGEN1.6\%20UNDP\%20GenderAnalysis\%20 toolkit.

Gómez, A. (2003). Colombia: el contexto de la desigualdad y la pobreza rural en los noventa. Cuadernos de Economía, I(1), 199-238.

González, A. (2008). Economía y sociedad: los retos del modelo económico. En Colectivo de autores, Antología del pensamiento económico (t. III). La Habana: Editorial Félix Varela.

González, F. (1999). Colombia, una nación fragmentada. Cuadernos Bakeaz, (36).

Grupo Faro. (2007). El enfoque de género en la asignación de los recursos: invirtiendo en un Ecuador más equitativo. Ecuador: Fondo de Desarrollo de las Naciones Unidas para la Mujer, Oficina para la Región Andina.

Hafner-Burty, E. y Pollack, M. (2002). Mainstreaming Gender in Global Governance. European
Journal of International Relations, VIII(3), 339-373.

Hoyos, L. M. (2012). El método neoconstitucional de la dignidad en el derecho privado: una aproximación a la igualdad de género en el ámbito de las relaciones familiares homoparentales. Revista de Derecho Privado, (47), 1-24.

Hoyos, L. M. (Enero-Junio, 2013). Derecho económico y género: alianza para la erradicación de los efectos adversos del desarrollo contra la mujer colombiana. Con-texto. Revista de Derecho y Economía, (39), 93-118.

Hoyos, L. M. y Álvarez, R. (2014). Mujer sorda y violencia basada en género: acciones que contribuyen al mejoramiento de la intervención en las ciudades de Bogotá, Medellín, Cali y Barranquilla (Colombia). En Proyecto de investigación del Instituto Nacional para Sordos -INSOR. Bogotá D. C.: INSOR.

Kalmanovitz, S. (2001). Las instituciones y el desarrollo económico en Colombia. Bogotá: Grupo Editorial Norma.

Kresalja Rosello, B. (2004). El constitucionalismo económico y las características del sistema económico del Perú. En el libro Homenaje a Jorge Avendaño Valdez. Lima: Ed. Fondo Editorial PUCP.

Layne, C. y Schwars, B. (1993). American Hegemony - Without an Enemy. Foreign Policy, (92), 5-23. 
López, C. (1994). Política económica y política social: una sola estrategia para la modernidad. Examen, (62).

Luna, L. (2003). Los movimientos de mujeres en América Latina y la renovación de la historia política. Santiago de Cali: La Manzana de la Discordia.

Medicina Legal, Profamilia y DANE. (2013). La mujer en cifras. Informe General (2012-2013). Bogotá: autores.

Mella Vázquez, M. (comp.). (1985). La izquierda europea. Análisis de la crisis de las ideologías de izquierda. Barcelona: Teide.

Moser, C. (1988). Gender Planning in the Third World: Meeting Practical and Strategic Gender. World Development, XVII(11), 1799-1825.

Nieto, A, y Monedero, C. (1977). Ideología y sociología del movimiento estudiantil. Madrid:

Ocampo, J. A. (2000). Cambio estructural, mercado laboral y distribución del ingreso: Colombia en la década de los noventa. Revista de la Cepal, (72), 53-78.

Olivera, J. (1981). Derecho económico, conceptos y problemas fundamentales. Buenos Aires: Ediciones Macchi.

Organización Internacional del Trabajo - оाт. (2008). Género y desarrollo. Obtenido de Organización Internacional del Trabajo: http:// www.ilo.org/global/topics/economic-and-so- cial-development/gender-and-development/ lang--es/index.htm

Overholt, C., Anderson, M., Cloud, K. y Austin, J. (1985). Gender Roles in Development Projects: Cases for Planners. West Hartford, CT: Kumarian Press.

Perry, G. (1972). Introducción al estudio de los planes de desarrollo para Colombia. Bogotá: Fundación para la Educación Superior y el Desarrollo.

Posner, R. (1993). What do Judges Maximize. Chicago: The Law School. The University of Chicago.

Presidencia de la República de Colombia. (2010). Misión para el empalme de las series de empleo, pobreza y desigualdad. Entrega de series actualizadas al Gobierno Nacional. Bogotá: DANE.

Programa de las Naciones Unidas para el Desarrollo. (2005). Gender Analysis. Versión libre de Evangelina García Prince. Obtenido de United Nations Development Programme: http://www.undp.org/content/dam/undp/library/gender/Institutional\%20Development/ TLGEN1.6\%20UNDP\%20GenderAnalysis\%20 toolkit.

Roldán Scott, J. (1992). El problema de la invisibilidad. En C. Ramos Escandón (comp.), Género e historia: la historiografía sobre la mujer. México DF: Universidad Autónoma Metropolitana. 
Sen, A. (1995). Nuevo examen de la desigualdad. (A. M. Bravo, Trad.) Madrid: Alianza Editorial, S. A.

Sen, A. (1998). Concepto de desarrollo humano. En Programa de los informes nacionales de desarrollo humano y objetivos del desarrollo del milenio. Guatemala: Programa de las Naciones Unidas para el Desarrollo.

Stiglitz, J. y Kumar Sah, R. (2002). Peasants Versus City-Dwellers: Taxation and the Burden of Economic Development. Oxford: Oxford University Press.

Strassman, D. (1999). Feminist Economics. En J. Peterson y M. Lewis (Edits.), The Elgar Companion to Feminist Economics. Cheltenham, UK: Edward Elgar Publishing Limited.
Tamayo, G.; Delso, A.; López, I.; Navarro, N. y Fdez-Layos, A. (2003). Género en la cooperación al desarrollo: una mirada a la desigualdad. Madrid: Acsur-Las Segovias.

Torres, I. (1972). María Cano mujer rebelde. Bogotá: Publicaciones de la Rosca.

Trubek, D. y Dezalay, Y. (1997). La internacionalización de los campos jurídicos y la creación de espacios transnacionales. Pensamiento Jurídico, (33-ss.).

Vela, B. (2005). El declive de los fundamentos económicos de la paz. Bogotá: Universidad Externado de Colombia.

Witker V., J. (1978). Antología de estudios sobre derecho económico. México: Universidad Nacional Autónoma de México. 